\title{
GREEN'S THEOREM FOR GENERALIZED FRACTIONAL DERIVATIVES
}

\author{
T. ODZIJEWICZ ${ }^{1}$, A. B. MALINOWSKA ${ }^{2}$, D. F. M. TORRES ${ }^{1}$
}

\begin{abstract}
We study three types of generalized partial fractional operators. An extension of Green's theorem, by considering partial fractional derivatives with more general kernels, is proved. New results are obtained, even in the particular case when the generalized operators are reduced to the standard partial fractional derivatives and fractional integrals in the sense of Riemann-Liouville or Caputo.
\end{abstract}

MSC 2010: Primary 26B20; Secondary 35R11

Key Words and Phrases: fractional calculus, generalized operators, Green's theorem

\section{INTRODUCTION}

In 1828, the English mathematician George Green (1793-1841), who up to his forties was working as a baker and a miller, published an essay where he introduced a formula connecting the line integral around a simple closed curve with a double integral. Within years, this result turned out to be useful in many fields of mathematics, physics and engineering [4, 6, 15, 17]. Generalizations of Green's theorem have chosen different directions, and are known as the Kelvin-Stokes and the Gauss-Ostrogradsky theorems.

In this paper, in contrast with previous works, we want to state a Green's theorem for generalized partial fractional derivatives. Notions of generalized fractional derivatives were introduced in [1, 8, and then developed in [11, 12]. A fractional version of the Green theorem has been already showed for Riemann-Liouville integrals and Caputo derivatives [18], and for fractional operators in the sense of Jumarie [3. However, generalized fractional operators have never been considered. Our result may be useful in the theory of fractional calculus (see, e.g., [7, 9, 14, 16]), in particular

This is a preprint of a paper whose final and definite form will appear at www.springerlink.com: Fract. Calc. Appl. Anal. 16 (2013), no. 1, in press. 
for the two-dimensional fractional calculus of variations, where the derivation of Euler-Lagrange equations uses, as a key step in the proof, Green's theorem [3, 5, 10, 13.

The paper is organized as follows. In Section 2 a common review of ordinary and partial generalized fractional calculus is given. Our results are then formulated and proved in Section 3, we show the two-dimensional integration by parts formula for generalized Riemann-Liouville partial fractional integrals (Theorem 3.1) and Green's theorem for generalized partial fractional derivatives (Theorem 3.2 ).

\section{BASIC Notions}

In this section we give definitions of generalized ordinary and partial fractional operators. By the choice of a certain kernel, these operators can be reduced to the standard fractional integrals and derivatives. For more on the subject, we refer the reader to $[1,2,8,11,12$.

\subsection{Generalized fractional operators.}

Definition 2.1 (Generalized fractional integral). The operator $K_{P}^{\alpha}$ is given by

$$
\left(K_{P}^{\alpha} f\right)(t):=p \int_{a}^{t} k_{\alpha}(t, \tau) f(\tau) d \tau+q \int_{t}^{b} k_{\alpha}(\tau, t) f(\tau) d \tau,
$$

where $P=\langle a, t, b, p, q\rangle$ is the parameter set ( $p$-set for brevity), $t \in[a, b]$, $p, q$ are real numbers, and $k_{\alpha}(t, \tau)$ is a kernel which may depend on $\alpha$. The operator $K_{P}^{\alpha}$ is referred as the operator $K$ (K-op for simplicity) of order $\alpha$ and $p$-set $P$.

TheOREM 2.1 (Theorem 2.3 of [11]). Let $k_{\alpha}$ be a difference kernel, i.e., $k_{\alpha}(t, \tau)=k_{\alpha}(t-\tau)$ and $k_{\alpha} \in L_{1}([a, b])$. Then, $K_{P}^{\alpha}: L_{1}([a, b]) \rightarrow L_{1}([a, b])$ is well defined, bounded and linear operator.

The $K$-op reduces to the classical left or right Riemann-Liouville fractional integral (see, e.g., [7, 14]) for a suitably chosen kernel $k_{\alpha}(t, \tau)$ and $p$-set $P$. Indeed, let $k_{\alpha}(t-\tau)=\frac{1}{\Gamma(\alpha)}(t-\tau)^{\alpha-1}$. If $P=\langle a, t, b, 1,0\rangle$, then

$$
\left(K_{P}^{\alpha} f\right)(t)=\frac{1}{\Gamma(\alpha)} \int_{a}^{t}(t-\tau)^{\alpha-1} f(\tau) d \tau=:\left({ }_{a} I_{t}^{\alpha} f\right)(t)
$$


is the left Riemann-Liouville fractional integral of order $\alpha$; if $P=\langle a, t, b, 0,1\rangle$, then

$$
\left(K_{P}^{\alpha} f\right)(t)=\frac{1}{\Gamma(\alpha)} \int_{t}^{b}(\tau-t)^{\alpha-1} f(\tau) d \tau=:\left({ }_{t} I_{b}^{\alpha} f\right)(t)
$$

is the right Riemann-Liouville fractional integral of order $\alpha$.

Definition 2.2 (Generalized Riemann-Liouville derivative). Let $P$ be a given parameter set. The operator $A_{P}^{\alpha}, 0<\alpha<1$, is defined for functions $f$ such that $K_{P}^{1-\alpha} f \in A C([a, b])$ by $A_{P}^{\alpha}:=\frac{d}{d t} \circ K_{P}^{1-\alpha}$, where $D$ denotes the standard derivative. We refer to $A_{P}^{\alpha}$ as operator $A$ ( $A$-op) of order $\alpha$ and $p$-set $P$.

Definition 2.3 (Generalized Caputo derivative). Let $P$ be a given parameter set. The operator $B_{P}^{\alpha}, \alpha \in(0,1)$, is defined for functions $f$ such that $f \in A C([a, b])$ by $B_{P}^{\alpha}:=K_{P}^{1-\alpha} \circ \frac{d}{d t}$ and is referred as the operator $B$ $(B$-op) of order $\alpha$ and $p$-set $P$.

Let $k_{1-\alpha}(t-\tau)=\frac{1}{\Gamma(1-\alpha)}(t-\tau)^{-\alpha}, \alpha \in(0,1)$. If $P=\langle a, t, b, 1,0\rangle$, then

$$
\left(A_{P}^{\alpha} f\right)(t)=\frac{1}{\Gamma(1-\alpha)} \frac{d}{d t} \int_{a}^{t}(t-\tau)^{-\alpha} f(\tau) d \tau=:\left({ }_{a} D_{t}^{\alpha} f\right)(t)
$$

is the standard left Riemann-Liouville fractional derivative of order $\alpha$ while

$$
\left(B_{P}^{\alpha} f\right)(t)=\frac{1}{\Gamma(1-\alpha)} \int_{a}^{t}(t-\tau)^{-\alpha} f^{\prime}(\tau) d \tau=:\left({ }_{a}^{C} D_{t}^{\alpha} f\right)(t)
$$

is the standard left Caputo fractional derivative of order $\alpha$; if $P=\langle a, t, b, 0,1\rangle$, then

$$
-\left(A_{P}^{\alpha} f\right)(t)=-\frac{1}{\Gamma(1-\alpha)} \frac{d}{d t} \int_{t}^{b}(\tau-t)^{-\alpha} f(\tau) d \tau=:\left({ }_{t} D_{b}^{\alpha} f\right)(t)
$$

is the standard right Riemann-Liouville fractional derivative of order $\alpha$ while

$$
-\left(B_{P}^{\alpha} f\right)(t)=-\frac{1}{\Gamma(1-\alpha)} \int_{t}^{b}(\tau-t)^{-\alpha} f^{\prime}(\tau) d \tau=:\left({ }_{t}^{C} D_{b}^{\alpha} f\right)(t)
$$

is the standard right Caputo fractional derivative of order $\alpha$. 
2.2. Generalized partial fractional operators. Let $\alpha$ be a real number from the interval $(0,1), \Delta_{n}=\left[a_{1}, b_{1}\right] \times \cdots \times\left[a_{n}, b_{n}\right], n \in \mathbb{N}$, be a subset of $\mathbb{R}^{n}$, $\mathbf{t}=\left(t_{1}, \ldots, t_{n}\right)$ be a point in $\Delta_{n}$ and $\mathbf{p}=\left(p_{1}, \ldots, p_{n}\right), \mathbf{q}=\left(q_{1}, \ldots, q_{n}\right) \in$ $\mathbb{R}^{n}$. Generalized partial fractional integrals and derivatives are a natural generalization of the corresponding one-dimensional generalized fractional integrals and derivatives.

Definition 2.4 (Generalized partial fractional integral). Let function $f=f\left(t_{1}, \ldots, t_{n}\right)$ be continuous on the set $\Delta_{n}$. The generalized partial Riemann-Liouville fractional integral of order $\alpha$ with respect to the $i$ th variable $t_{i}$ is given by

$$
\begin{aligned}
& \left(K_{P_{t_{i}}}^{\alpha} f\right)(\mathbf{t}):=p_{i} \int_{a_{i}}^{t_{i}} k_{\alpha}\left(t_{i}, \tau\right) f\left(t_{1}, \ldots, t_{i-1}, \tau, t_{i+1}, \ldots, t_{n}\right) d \tau \\
& \quad+q_{i} \int_{t_{i}}^{b_{i}} k_{\alpha}\left(\tau, t_{i}\right) f\left(t_{1}, \ldots, t_{i-1}, \tau, t_{i+1}, \ldots, t_{n}\right) d \tau,
\end{aligned}
$$

where $P_{t_{i}}=\left\langle a_{i}, t_{i}, b_{i}, p_{i}, q_{i}\right\rangle$. We refer to $K_{P_{t_{i}}}^{\alpha}$ as the partial operator $K$ (partial $K$-op) of order $\alpha$ and $p$-set $P_{t_{i}}$.

Definition 2.5 (Generalized partial Riemann-Liouville derivatives). Let $P_{t_{i}}=\left\langle a_{i}, t_{i}, b_{i}, p_{i}, q_{i}\right\rangle$ and $K_{P_{t_{i}}}^{1-\alpha} f \in C^{1}\left(\Delta_{n}\right)$. The generalized partial Riemann-Liouville fractional derivative of order $\alpha$ with respect to the $i$ th variable $t_{i}$ is given by

$$
\begin{aligned}
\left(A_{P_{t_{i}}}^{\alpha} f\right)(\mathbf{t}) & :=\left(\frac{\partial}{\partial t_{i}} \circ K_{P_{t_{i}}}^{1-\alpha} f\right)(\mathbf{t}) \\
= & \frac{\partial}{\partial t_{i}}\left(p_{i} \int_{a_{i}}^{t_{i}} k_{1-\alpha}\left(t_{i}, \tau\right) f\left(t_{1}, \ldots, t_{i-1}, \tau, t_{i+1}, \ldots, t_{n}\right) d \tau\right. \\
& \left.\quad+q_{i} \int_{t_{i}}^{b_{i}} k_{1-\alpha}\left(\tau, t_{i}\right) f\left(t_{1}, \ldots, t_{i-1}, \tau, t_{i+1}, \ldots, t_{n}\right) d \tau\right) .
\end{aligned}
$$

The operator $A_{P_{t_{i}}}^{\alpha}$ is referred as the partial operator $A$ (partial $A$-op) of order $\alpha$ and $p$-set $P_{t_{i}}$.

Definition 2.6 (Generalized partial Caputo derivative). Let $P_{t_{i}}=$ $\left\langle a_{i}, t_{i}, b_{i}, p_{i}, q_{i}\right\rangle$ and $f \in C^{1}\left(\Delta_{n}\right)$. The generalized partial Caputo fractional 
derivative of order $\alpha$ with respect to the $i$ th variable $t_{i}$ is given by

$$
\begin{aligned}
\left(B_{P_{t_{i}}}^{\alpha} f\right)(\mathbf{t}) & :=\left(K_{P_{t_{i}}}^{1-\alpha} \circ \frac{\partial}{\partial t_{i}} f\right)(\mathbf{t}) \\
= & p_{i} \int_{a_{i}}^{t_{i}} k_{1-\alpha}\left(t_{i}, \tau\right) \frac{\partial}{\partial \tau} f\left(t_{1}, \ldots, t_{i-1}, \tau, t_{i+1}, \ldots, t_{n}\right) d \tau \\
& \quad+q_{i} \int_{t_{i}}^{b_{i}} k_{1-\alpha}\left(\tau, t_{i}\right) \frac{\partial}{\partial \tau} f\left(t_{1}, \ldots, t_{i-1}, \tau, t_{i+1}, \ldots, t_{n}\right) d \tau
\end{aligned}
$$

and is referred as the partial operator $B$ (partial $B$-op) of order $\alpha$ and $p$-set $P_{t_{i}}$.

Similarly as in the one-dimensional case [1, 11, 12, the generalized partial operators $K, A$ and $B$ here introduced give the standard partial fractional integrals and derivatives for particular kernels and $p$-sets. The leftand right-sided Riemann-Liouville partial fractional integrals with respect to the $i$ th variable $t_{i}$ are obtained by choosing the kernel

$$
k_{\alpha}\left(t_{i}, \tau\right)=\frac{1}{\Gamma(\alpha)}\left(t_{i}-\tau\right)^{\alpha-1}
$$

and $p$-sets $L_{t_{i}}=\left\langle a_{i}, t_{i}, b_{i}, 1,0\right\rangle$ and $R_{t_{i}}=\left\langle a_{i}, t_{i}, b_{i}, 0,1\right\rangle$, respectively:

$$
\begin{aligned}
\left(a_{i} I_{t_{i}}^{\alpha} f\right)(\mathbf{t}) & =\left(K_{L_{t_{i}}}^{\alpha} f\right)(\mathbf{t}) \\
& =\frac{1}{\Gamma(\alpha)} \int_{a_{i}}^{t_{i}}\left(t_{i}-\tau\right)^{\alpha-1} f\left(t_{1}, \ldots, t_{i-1}, \tau, t_{i+1}, \ldots, t_{n}\right) d \tau \\
\left(t_{i} I_{b_{i}}^{\alpha} f\right)(\mathbf{t}) & =\left(K_{R_{t_{i}}}^{\alpha} f\right)(\mathbf{t}) \\
& =\frac{1}{\Gamma(\alpha)} \int_{t_{i}}^{b_{i}}\left(\tau-t_{i}\right)^{\alpha-1} f\left(t_{1}, \ldots, t_{i-1}, \tau, t_{i+1}, \ldots, t_{n}\right) d \tau .
\end{aligned}
$$

The standard left- and right-sided partial Riemann-Liouville and Caputo fractional derivatives with respect to the $i$ th variable $t_{i}$ are obtained with the choice of kernel $k_{1-\alpha}\left(t_{i}, \tau\right)=\frac{1}{\Gamma(1-\alpha)}\left(t_{i}-\tau\right)^{-\alpha}$ : if $P_{t_{i}}=\left\langle a_{i}, t_{i}, b_{i}, 1,0\right\rangle$, 
then

$$
\begin{aligned}
\left(a_{i} D_{t_{i}}^{\alpha} f\right)(\mathbf{t}) & =\left(A_{P_{t_{i}}}^{\alpha} f\right)(\mathbf{t}) \\
& =\frac{1}{\Gamma(1-\alpha)} \frac{\partial}{\partial t_{i}} \int_{a_{i}}^{t_{i}}\left(t_{i}-\tau\right)^{-\alpha} f\left(t_{1}, \ldots, t_{i-1}, \tau, t_{i+1}, \ldots, t_{n}\right) d \tau
\end{aligned}
$$

and

$$
\begin{aligned}
\left({ }_{a_{i}}^{C} D_{t_{i}}^{\alpha} f\right)(\mathbf{t}) & =\left(B_{P_{t_{i}}}^{\alpha} f\right)(\mathbf{t}) \\
& =\frac{1}{\Gamma(1-\alpha)} \int_{a_{i}}^{t_{i}}\left(t_{i}-\tau\right)^{-\alpha} \frac{\partial}{\partial \tau} f\left(t_{1}, \ldots, t_{i-1}, \tau, t_{i+1}, \ldots, t_{n}\right) d \tau
\end{aligned}
$$

if $P_{t_{i}}=\left\langle a_{i}, t_{i}, b_{i}, 0,1\right\rangle$, then

$$
\begin{aligned}
\left(t_{i} D_{b_{i}}^{\alpha} f\right)(\mathbf{t}) & =-\left(A_{P_{t_{i}}}^{\alpha} f\right)(\mathbf{t}) \\
& =-\frac{1}{\Gamma(1-\alpha)} \frac{\partial}{\partial t_{i}} \int_{t_{i}}^{b_{i}}\left(\tau-t_{i}\right)^{-\alpha} f\left(t_{1}, \ldots, t_{i-1}, \tau, t_{i+1}, \ldots, t_{n}\right) d \tau
\end{aligned}
$$

and

$$
\begin{aligned}
\left(\begin{array}{l}
C \\
t_{i}
\end{array} D_{b_{i}}^{\alpha} f\right)(\mathbf{t}) & =-\left(B_{P_{t_{i}}}^{\alpha} f\right)(\mathbf{t}) \\
& =-\frac{1}{\Gamma(1-\alpha)} \int_{t_{i}}^{b_{i}}\left(\tau-t_{i}\right)^{-\alpha} \frac{\partial}{\partial \tau} f\left(t_{1}, \ldots, t_{i-1}, \tau, t_{i+1}, \ldots, t_{n}\right) d \tau
\end{aligned}
$$

Remark 2.1. In Definitions 2.4, 2.5 and 2.6, all the variables, except $t_{i}$, are kept fixed. That choice of fixed values determines a function $f_{t_{1}, \ldots, t_{i-1}, t_{i+1}, \ldots, t_{n}}:\left[a_{i}, b_{i}\right] \rightarrow \mathbb{R}$ of one variable $t_{i}:$

$$
f_{t_{1}, \ldots, t_{i-1}, t_{i+1}, \ldots, t_{n}}\left(t_{i}\right)=f\left(t_{1}, \ldots, t_{i-1}, t_{i}, t_{i+1}, \ldots, t_{n}\right) .
$$

By Definitions 2.1, 2.2, 2.3 and 2.4, 2.5, 2.6, we have

$$
\begin{aligned}
& \left(K_{P_{t_{i}}}^{\alpha} f_{t_{1}, \ldots, t_{i-1}, t_{i+1}, \ldots, t_{n}}\right)\left(t_{i}\right)=\left(K_{P_{t_{i}}}^{\alpha} f\right)\left(t_{1}, \ldots, t_{i-1}, t_{i}, t_{i+1}, \ldots, t_{n}\right), \\
& \left(A_{P_{t_{i}}}^{\alpha} f_{t_{1}, \ldots, t_{i-1}, t_{i+1}, \ldots, t_{n}}\right)\left(t_{i}\right)=\left(A_{P_{t_{i}}}^{\alpha} f\right)\left(t_{1}, \ldots, t_{i-1}, t_{i}, t_{i+1}, \ldots, t_{n}\right), \\
& \left(B_{P_{t_{i}}}^{\alpha} f_{t_{1}, \ldots, t_{i-1}, t_{i+1}, \ldots, t_{n}}\right)\left(t_{i}\right)=\left(B_{P_{t_{i}}}^{\alpha} f\right)\left(t_{1}, \ldots, t_{i-1}, t_{i}, t_{i+1}, \ldots, t_{n}\right) .
\end{aligned}
$$

Therefore, as in the classical integer order case, computation of partial generalized fractional operators is reduced to the computation of one-variable generalized fractional operators. 


\section{Green's Theorem for Generalized Fractional Derivatives}

Definition 3.1 (Dual $p$-set). Let $P_{t_{i}}=\left\langle a_{i}, t_{i}, b_{i}, p_{i}, q_{i}\right\rangle, i \in \mathbb{N}$. We denote by $P_{t_{i}}^{*}$ the $p$-set $P_{t_{i}}^{*}=\left\langle a_{i}, t_{i}, b_{i}, q_{i}, p_{i}\right\rangle$ and call it the dual of $P_{t_{i}}$.

Theorem 3.1 (Generalized 2D Integration by Parts). Let $\alpha \in(0,1)$, $P_{t_{i}}=\left\langle a_{i}, t_{i}, b_{i}, p_{i}, q_{i}\right\rangle$ be a parameter set, and $k_{\alpha}$ be a difference kernel, i.e., $k_{\alpha}\left(t_{i}, \tau\right)=k_{\alpha}\left(t_{i}-\tau\right)$ such that $k_{\alpha} \in L_{1}\left(\left[0, b_{i}-a_{i}\right]\right), i=1,2$. If $f, g, \eta_{1}, \eta_{2} \in C\left(\Delta_{2}\right)$, then the generalized partial fractional integrals satisfy the following identity:

$$
\begin{aligned}
\int_{a_{1}}^{b_{1}} \int_{a_{2}}^{b_{2}}\left[g(\boldsymbol{t})\left(K_{P_{t_{1}}}^{\alpha} \eta_{1}\right)(\boldsymbol{t})+f(\boldsymbol{t})\left(K_{P_{t_{2}}}^{\alpha} \eta_{2}\right)(\boldsymbol{t})\right] d t_{2} d t_{1} \\
=\int_{a_{1}}^{b_{1}} \int_{a_{2}}^{b_{2}} \eta_{1}(\boldsymbol{t})\left[\left(K_{P_{t_{1}}^{*}}^{\alpha} g\right)(\boldsymbol{t})\right]+\eta_{2}(\boldsymbol{t})\left[\left(K_{P_{t_{2}}^{*}}^{\alpha} f\right)(\boldsymbol{t})\right] d t_{2} d t_{1}
\end{aligned}
$$

where $P_{t_{i}}^{*}$ is the dual of $P_{t_{i}}, i=1,2$.

P r o o f. Define

$$
F_{1}(\mathbf{t}, \tau):= \begin{cases}\left|p_{1} k_{\alpha}\left(t_{1}-\tau\right)\right| \cdot|g(\mathbf{t})| \cdot\left|\eta_{1}\left(\tau, t_{2}\right)\right| & \text { if } \tau \leq t_{1} \\ \left|q_{1} k_{\alpha}\left(\tau-t_{1}\right)\right| \cdot|g(\mathbf{t})| \cdot\left|\eta_{1}\left(\tau, t_{2}\right)\right| & \text { if } \tau>t_{1}\end{cases}
$$

for all $(\mathbf{t}, \tau) \in\left[a_{1}, b_{1}\right] \times\left[a_{2}, b_{2}\right] \times\left[a_{1}, b_{1}\right]$ and

$$
F_{2}(\mathbf{t}, \tau):= \begin{cases}\left|p_{2} k_{\alpha}\left(t_{2}-\tau\right)\right| \cdot|f(\mathbf{t})| \cdot\left|\eta_{2}\left(t_{1}, \tau\right)\right| & \text { if } \tau \leq t_{2} \\ \left|q_{2} k_{\alpha}\left(\tau-t_{2}\right)\right| \cdot|f(\mathbf{t})| \cdot\left|\eta_{2}\left(t_{1}, \tau\right)\right| & \text { if } \tau>t_{2}\end{cases}
$$

for all $(\mathbf{t}, \tau) \in\left[a_{1}, b_{1}\right] \times\left[a_{2}, b_{2}\right] \times\left[a_{2}, b_{2}\right]$. Since $f, g$ and $\eta_{i}, i=1,2$, are continuous functions on $\Delta_{2}$, they are bounded on $\Delta_{2}$. Hence, there exist real numbers $C_{1}, C_{2}, C_{3}, C_{4}>0$ such that

$$
|f(\mathbf{t})| \leq C_{1}, \quad|g(\mathbf{t})| \leq C_{2}, \quad\left|\eta_{1}(\mathbf{t})\right| \leq C_{3}, \quad\left|\eta_{2}(\mathbf{t})\right| \leq C_{4}
$$


for all $\mathbf{t} \in \Delta_{2}$. Therefore,

$$
\begin{aligned}
& \int_{a_{1}}^{b_{1}} \int_{a_{2}}^{b_{2}} \int_{a_{1}}^{b_{1}} F_{1}(\mathbf{t}, \tau) d t_{1} d t_{2} d \tau+\int_{a_{1}}^{b_{1}} \int_{a_{2}}^{b_{2}} \int_{a_{2}}^{b_{2}} F_{2}(\mathbf{t}, \tau) d t_{2} d \tau d t_{1} \\
&=\int_{a_{1}}^{b_{1}}\left(\int _ { a _ { 2 } } ^ { b _ { 2 } } \left(\int_{\tau}^{b_{1}}\left|p_{1} k_{\alpha}\left(t_{1}-\tau\right)\right| \cdot|g(\mathbf{t})| \cdot\left|\eta_{1}\left(\tau, t_{2}\right)\right| d t_{1}\right.\right. \\
&\left.\left.+\int_{a_{1}}^{\tau}\left|q_{1} k_{\alpha}\left(\tau-t_{1}\right)\right| \cdot|g(\mathbf{t})| \cdot\left|\eta_{1}\left(\tau, t_{2}\right)\right| d t_{1}\right) d t_{2}\right) d \tau \\
&+\int_{a_{1}}^{b_{1}}\left(\int _ { a _ { 2 } } ^ { b _ { 2 } } \left(\int_{\tau}^{b_{2}}\left|p_{2} k_{\alpha}\left(t_{2}-\tau\right)\right| \cdot|f(\mathbf{t})| \cdot\left|\eta_{2}\left(t_{1}, \tau\right)\right| d t_{2}\right.\right. \\
&\left.\left.+\int_{a_{2}}^{\tau}\left|q_{2} k_{\alpha}\left(\tau-t_{2}\right)\right| \cdot|f(\mathbf{t})| \cdot\left|\eta_{2}\left(t_{1}, \tau\right)\right| d t_{2}\right) d \tau\right) d t_{1} \\
& \leq C_{2} C_{3}\left[\int _ { a _ { 1 } } ^ { b _ { 1 } } \left(\int _ { a _ { 2 } } ^ { b _ { 2 } } \left(\int_{\tau}^{b_{1}}\left|p_{1} k_{\alpha}\left(t_{1}-\tau\right)\right| d t_{1}\right.\right.\right. \\
&\left.\left.\left.+\int_{a_{1}}^{\tau}\left|q_{1} k_{\alpha}\left(\tau-t_{1}\right)\right| d t_{1}\right) d t_{2}\right) d \tau\right] \\
&+C_{1} C_{4}\left[\int _ { a _ { 1 } } ^ { b _ { 1 } } \left(\int _ { a _ { 2 } } ^ { b _ { 2 } } \left(\int_{\tau}^{b_{2}}\left|p_{2} k_{\alpha}\left(t_{2}-\tau\right)\right| d t_{2}\right.\right.\right. \\
&\left.\left.\left.+\int_{\tau}^{b_{2}}\left|q_{2} k_{\alpha}\left(\tau-t_{2}\right)\right| d t_{2}\right) d \tau\right) d t_{1}\right] \\
&+C_{2} C_{3}\left(\left|p_{1}\right|+\left|q_{1}\right|\right)\left\|k_{\alpha}\right\|\left(b_{2}-a_{2}\right)\left(b_{1}-a_{1}\right) \\
&< C_{1} C_{3}\left[\int _ { a _ { 1 } } ^ { b _ { 1 } } \left(\int _ { a _ { 2 } } ^ { b _ { 2 } } \left(\int_{0}^{b_{1}-a_{1}}\left|p_{1} k_{\alpha}\left(u_{1}\right)\right| d u_{1}\right.\right.\right. \\
&\left.\left.\left.+\int_{0}^{b_{1}-a_{1}}\left|q_{1} k_{\alpha}\left(u_{1}\right)\right| d u_{1}\right) d t_{2}\right) d \tau\right] \\
&+C_{1} C_{4}\left[\int _ { a _ { 1 } } ^ { b _ { 1 } } \left(\int _ { a _ { 2 } } ^ { b _ { 2 } } \left(\int_{0}^{b_{2}-a_{2}}\left|p_{2} k_{\alpha}\left(u_{2}\right)\right| d u_{2}\right.\right.\right. \\
&\left.\left.+q_{2} k_{\alpha}\left(u_{2}\right) \mid d u_{2}\right) d \tau\right) d t_{1} \|\left(b_{2}-a_{2}\right)\left(b_{1}-a_{1}\right) \\
&\left.+\int_{0}\right)
\end{aligned}
$$

Hence, we can use Fubini's theorem to change the order of integration in the iterated integrals:

$$
\int_{a_{1}}^{b_{1}} \int_{a_{2}}^{b_{2}}\left[g(\mathbf{t})\left(K_{P_{t_{1}}}^{\alpha} \eta_{1}\right)(\mathbf{t})+f(\mathbf{t})\left(K_{P_{t_{2}}}^{\alpha} \eta_{2}\right)(\mathbf{t})\right] d t_{2} d t_{1}
$$




$$
\begin{aligned}
\text { GREEN'S THEOREM FOR GENERALIZED FRACTIONAL } \ldots \\
=\int_{a_{1}}^{b_{1}} \int_{a_{2}}^{b_{2}}\left[g ( \mathbf { t } ) \left(p_{1} \int_{a_{1}}^{t_{1}} k_{\alpha}\left(t_{1}-\tau\right) \eta_{1}\left(\tau, t_{2}\right) d \tau\right.\right. \\
\left.\quad+q_{1} \int_{t_{1}}^{b_{1}} k_{\alpha}\left(\tau-t_{1}\right) \eta_{1}\left(\tau, t_{2}\right) d \tau\right) \\
+f(\mathbf{t})\left(p_{2} \int_{a_{2}}^{t_{2}} k_{\alpha}\left(t_{2}-\tau\right) \eta_{2}\left(t_{1}, \tau\right) d \tau\right. \\
\left.\left.+q_{2} \int_{t_{2}}^{b_{2}} k_{\alpha}\left(\tau-t_{2}\right) \eta_{2}\left(t_{1}, \tau\right) d \tau\right)\right] d t_{2} d t_{1} \\
=\int_{a_{2}}^{b_{2}}\left(\int _ { a _ { 1 } } ^ { b _ { 1 } } \eta _ { 1 } ( \tau , t _ { 2 } ) \left(p_{1} \int_{\tau}^{b_{1}} k_{\alpha}\left(t_{1}-\tau\right) g(\mathbf{t}) d t_{1}\right.\right. \\
\left.\left.+q_{1} \int_{a_{1}}^{\tau} k_{\alpha}\left(\tau-t_{1}\right) g(\mathbf{t}) d t_{1}\right) d \tau\right) d t_{2} \\
+\int_{a_{1}}^{b_{1}}\left(\int _ { a _ { 2 } } ^ { b _ { 2 } } \eta _ { 2 } ( t _ { 1 } , \tau ) \left(p_{2} \int_{\tau}^{b_{2}} k_{\alpha}\left(t_{2}-\tau\right) f(\mathbf{t}) d t_{2}\right.\right. \\
\left.\left.+q_{2} \int_{a_{2}}^{\tau} k_{\alpha}\left(\tau-t_{2}\right) f(\mathbf{t}) d t_{2}\right) d \tau\right) d t_{1} \\
=\int_{a_{1}}^{b_{1}} \int_{a_{2}}^{b_{2}} \eta_{1}\left(\tau, t_{2}\right)\left(K_{P_{1}^{*}}^{\alpha} g\right)\left(\tau, t_{2}\right) d t_{2} d \tau \\
+\int_{a_{1}}^{b_{1}} \int_{a_{2}}^{b_{2}} \eta_{2}\left(t_{1}, \tau\right)\left(K_{P_{t_{2}^{*}}}^{\alpha} f\right)\left(t_{1}, \tau\right) d \tau d t_{1} .
\end{aligned}
$$

We are now in conditions to state and prove the main result of the paper: the Green theorem for generalized fractional derivatives.

Theorem 3.2 (Generalized Green's Theorem). Let $0<\alpha<1$ and $f, g, \eta \in C^{1}\left(\Delta_{2}\right)$. Let $k_{\alpha}$ be a difference kernel, i.e., $k_{\alpha}\left(t_{i}, \tau\right)=k_{\alpha}\left(t_{i}-\tau\right)$ such that $k_{\alpha} \in L_{1}\left(\left[0, b_{i}-a_{i}\right]\right), i=1,2$, and $K_{P_{t_{1}}^{*}}^{1-\alpha} g, K_{P_{t_{2}}^{*}}^{1-\alpha} f \in C^{1}\left(\Delta_{2}\right)$. Then, the following formula holds:

$$
\begin{aligned}
& \int_{a_{1}}^{b_{1}} \int_{a_{2}}^{b_{2}} {\left[g(\boldsymbol{t})\left(B_{P_{t_{1}}}^{\alpha} \eta\right)(\boldsymbol{t})+f(\boldsymbol{t})\left(B_{P_{t_{2}}}^{\alpha} \eta\right)(\boldsymbol{t})\right] d t_{2} d t_{1} } \\
&=-\int_{a_{1}}^{b_{1}} \int_{a_{2}}^{b_{2}} \eta(\boldsymbol{t})\left[\left(A_{P_{t_{1}}^{*}}^{\alpha} g\right)(\boldsymbol{t})+\left(A_{P_{t_{2}}^{*}}^{\alpha} f\right)(\boldsymbol{t})\right] d t_{2} d t_{1} \\
& \quad+\oint_{\partial \Delta_{2}} \eta(\boldsymbol{t})\left[\left(K_{P_{t_{1}}^{*}}^{1-\alpha} g\right)(\boldsymbol{t}) d t_{2}-\left(K_{P_{t_{2}}^{*}}^{1-\alpha} f\right)(\boldsymbol{t}) d t_{1}\right] .
\end{aligned}
$$


P r o o f. By the definition of generalized partial Caputo fractional derivative, Theorem 3.1, and the standard Green's theorem, one has

$$
\begin{aligned}
\int_{a_{1}}^{b_{1}} & \int_{a_{2}}^{b_{2}}\left[g(\mathbf{t})\left(B_{P_{t_{1}}}^{\alpha} \eta\right)(\mathbf{t})+f(\mathbf{t})\left(B_{P_{t_{2}}}^{\alpha} \eta\right)(\mathbf{t})\right] d t_{2} d t_{1} \\
= & \int_{a_{1}}^{b_{1}} \int_{a_{2}}^{b_{2}}\left[g(\mathbf{t})\left(K_{P_{t_{1}}}^{1-\alpha} \frac{\partial}{\partial t_{1}} \eta\right)(\mathbf{t})+f(\mathbf{t})\left(K_{P_{t_{2}}}^{1-\alpha} \frac{\partial}{\partial t_{2}} \eta\right)(\mathbf{t})\right] d t_{2} d t_{1} \\
= & \int_{a_{1}}^{b_{1}} \int_{a_{2}}^{b_{2}}\left[\frac{\partial}{\partial t_{1}} \eta(\mathbf{t})\left(K_{P_{t_{1}}^{*}}^{1-\alpha} g\right)(\mathbf{t})+\frac{\partial}{\partial t_{2}} \eta(\mathbf{t})\left(K_{P_{t_{2}}^{*}}^{1-\alpha} f\right)(\mathbf{t})\right] d t_{2} d t_{1} \\
= & -\int_{a_{1}}^{b_{1}} \int_{a_{2}}^{b_{2}} \eta(\mathbf{t})\left[\frac{\partial}{\partial t_{1}}\left(K_{P_{t_{1}^{*}}^{*}}^{1-\alpha} g\right)(\mathbf{t})+\frac{\partial}{\partial t_{2}}\left(K_{P_{t_{2}}^{*}}^{1-\alpha} f\right)(\mathbf{t})\right] d t_{2} d t_{1} \\
& +\oint_{\partial \Delta_{2}} \eta(\mathbf{t})\left[\left(K_{P_{t_{1}}^{*}}^{1-\alpha} g\right)(\mathbf{t}) d t_{2}-\left(K_{P_{t_{2}}^{*}}^{1-\alpha} f\right)(\mathbf{t}) d t_{1}\right] .
\end{aligned}
$$

Corollary 3.1. Let $0<\alpha<1$ and $f, g, \eta \in C^{1}\left(\Delta_{2}\right)$. If $\left({ }_{t_{1}} I_{b_{1}}^{1-\alpha} g\right)(\boldsymbol{t})$ and $\left({ }_{t} I_{b_{2}}^{1-\alpha} f\right)(\boldsymbol{t})$ are continuously differentiable on the rectangle $\Delta_{2}$, then

$$
\begin{aligned}
& \int_{a_{1}}^{b_{1}} \int_{a_{2}}^{b_{2}}\left[g(\boldsymbol{t})\left({ }_{a_{1}}^{C} D_{t_{1}}^{\alpha} \eta\right)(\boldsymbol{t})+f(\boldsymbol{t})\left({ }_{a_{2}}^{C} D_{t_{2}}^{\alpha} \eta\right)(\boldsymbol{t})\right] d t_{2} d t_{1} \\
& =\int_{a_{1}}^{b_{1}} \int_{a_{2}}^{b_{2}} \eta(\boldsymbol{t})\left[\left(t_{1} D_{b_{1}}^{\alpha} g\right)(\boldsymbol{t})+\left({ }_{t_{2}} D_{b_{2}}^{\alpha} f\right)(\boldsymbol{t})\right] d t_{2} d t_{1} \\
& +\oint_{\partial \Delta_{2}} \eta(\boldsymbol{t})\left[\left(t_{1} I_{b_{1}}^{1-\alpha} g\right)(\boldsymbol{t}) d t_{2}-\left(t_{2} I_{b_{2}}^{1-\alpha} f\right)(\boldsymbol{t}) d t_{1}\right] .
\end{aligned}
$$

\section{ACKNOWLEDGEMENTS}

This work received The Grunwald-Letnikov Award: Best Student Paper (theory), at the 2012 Symposium on Fractional Differentiation and Its Applications (FDA'2012), May 16, 2012, Hohai University, Nanjing. It was supported by FEDER funds through COMPETE (Operational Program Factors of Competitiveness) and by Portuguese funds through the Center for Research and Development in Mathematics and Applications (CIDMA) and the Portuguese Foundation for Science and Technology (FCT), within project PEst-C/MAT/UI4106/2011 with COMPETE number FCOMP-010124-FEDER-022690. Odzijewicz was also supported by FCT under Ph.D. fellowship SFRH/BD/33865/2009; Malinowska by Białystok University of Technology grant S/WI/02/2011; and Torres by FCT through the project PTDC/MAT/113470/2009. 


\section{REFERENCES}

[1] O.P. Agrawal, Generalized variational problems and Euler-Lagrange equations, Comput. Math. Appl. 59, No 5 (2010), 1852-1864.

[2] O.P. Agrawal, Some generalized fractional calculus operators and their applications in integral equations, Fract. Calc. Appl. Anal. 15, No 4 (2012), 700-711.

[3] R. Almeida, A.B. Malinowska, D.F.M. Torres, A fractional calculus of variations for multiple integrals with application to vibrating string, $J$. Math. Phys. 51 (2010), 033503, 12 pp. arXiv:1001.2722

[4] D.L. Colton, R. Kress, Inverse acoustic and electromagnetic scattering theory, Second Edition, Springer (1998).

[5] J. Cresson, Fractional embedding of differential operators and Lagrangian systems, J. Math. Phys. 48 (2007), 033504, 34 pp. arXiv:math/0605752

[6] G.G. Emch, C. Liu, The logic and thermostatical physics, SpringerVerlag, New York (2002).

[7] A.A. Kilbas, H.M. Srivastava, J.J. Trujillo, Theory and applications of fractional differential equations, volume 204 of North-Holland Mathematics Studies, Elsevier Science B.V., Amsterdam (2006).

[8] V. Kiryakova, Generalized fractional calculus and applications, Pitman Research Notes in Mathematics Series, 301, Longman Sci. Tech., Harlow (1994).

[9] M. Klimek, On solutions of linear fractional differential equations of a variational type, The Publishing Office of Czenstochowa University of Technology, Czestochowa (2009).

[10] A.B. Malinowska, D.F.M. Torres, Introduction to the fractional calculus of variations, Imperial College Press, London \& World Scientific Publishing, Singapore (2012).

[11] T. Odzijewicz, A.B. Malinowska, D.F.M. Torres, Generalized fractional calculus with applications to the calculus of variations, Comput. Math. Appl. 64 (2012), no. 10, 3351-3366. arXiv:1201.5747

[12] T. Odzijewicz, A.B. Malinowska, D.F.M. Torres, Fractional calculus of variations in terms of a generalized fractional integral with applications to physics, Abstr. Appl. Anal. 2012 (2012), 871912, 24 pp. arXiv:1203.1961

[13] T. Odzijewicz, D.F.M. Torres, Fractional calculus of variations for double integrals, Balkan J. Geom. Appl. 16, No 2 (2011), 102-113. arXiv:1102.1337

[14] I. Podlubny, Fractional differential equations, Mathematics in Science and Engineering, 198, Academic Press, San Diego, CA (1999). 
[15] S. Russenschuck, Field Computation for accelerator magnets: analytical and numerical methods for electromagnetic design and optimization, Wiley-VCH Verlag GmbH and Co. KGaA, Weinheim (2010).

[16] S.G. Samko, A.A. Kilbas, O.I. Marichev, Fractional integrals and derivatives, translated from the 1987 Russian original, Gordon and Breach, Yverdon (1993).

[17] C.H. Sherman, J.L. Butler, Transducers and arrays for underwater sound, Springer-Verlag (2007).

[18] V.E. Tarasov, Fractional vector calculus and fractional Maxwell's equations, Ann. Physics 323, No 11 (2008), 2756-2778.

${ }^{1}$ Center for Research and Development in Mathematics and Applications Department of Mathematics

University of Aveiro 3810-193 Aveiro, PORTUGAL

e-mail: tatianao@ua.pt,delfim@ua.pt

Received: July 09, 2012

${ }^{2}$ Faculty of Computer Science

Biatystok University of Technology

15-351 Biatystok, POLAND

e-mail: a.malinowska@pb.edu.pl 